\title{
Radio, Millimeter and Infrared Observations of the Local Hot Bubble and Its Environment
}

\author{
Ulrich Mebold, Jürgen Kerp and Peter M.W. Kalberla
}

Radioastronomisches Institut der Universität Bonn, Auf dem Hügel 71, D-53121 Bonn, Germany

\begin{abstract}
We present a definition of the local void of neutral gas from observations in the radio frequency window. We question the concept of the Local Bubble in sense of a more or less spherical volume which is surrounded by a shell of denser gas. The concept of the Local Bubble is challenged by the discovery of numerous neutral, dense clouds inside the local void. The search for a "shell" around the suspected Bubble has resulted only in inconclusive findings so far. The sample of high latitude molecular clouds illustrates the situation particularly well. The statistical properties of their spatial distribution, e.g. the mean distance, seem to fit very nicely to the spatial extent of the Local Bubble. But a more detailed investigation shows that the concept of a bubble - in particular an expanding bubble - is not supported. We suggest that the local void is nothing more than a typical place in an interarm region of our Galaxy.

Finally, a discussion of the high latitude boundary of the local void does not give strong evidence for the concept of a bubble, that has once been in rapid expansion and is still showing signs of interaction with its environment. However, indications for interactions of IVCs or HVCs with their surroundings are found. These hint at the presence of a gaseous disk which is much more vertically extended than previously believed, or at a Galactic wind which may be blowing from the Galactic neighbourhood of the Sun.
\end{abstract}

\section{Discovery of the Local Void, Some History}

A void of neutral material in the interstellar medium (ISM) around the Sun was discovered by Frisch \& York (1983) in studies of nearby early type stars. In 1968 Bowyer, Field and Mack discovered the soft X-ray background radiation. On the basis of a new extensive data-set McCammon et al. (1983) proposed that all of the observed soft $\mathrm{X}$-ray radiation was emitted from within the local void of neutral matter. Their model of a Local Hot Bubble (LHB) was consistent with all the observations available until the launch of ROSAT (McCammon \& Sanders 1990). The effect of the local void on the H I gas has been known for many years. The $\mathrm{H}$ I column densities projected vertically onto the Galactic plane show a depression of about $30 \%$ right at the location of the Sun (see Fig. 1). The depression derived at $90^{\circ}$ and $z_{0}=400 \mathrm{pc}$ is about $40 \%$ on the north side of the Galactic plane and about $30 \%$ on the southern galactic sky. This difference shows that local void is more prominent in the northern Galactic plane then in the southern. The plots for different 
sections show the assymetry of the local void with respect to the Sun, in particular its large extension towards the third galactic quadrant. In fact this assymetry hints at an interpretation of the local void as a feature which is related to the local interarm region, the region between the Sagittarius and the Perseus spiral arms of our Galaxy. If this interpretation is correct, the solar surroundings were nothing special but a typical place in an interarm region of our Galaxy. In contrast to this view Snowden et al. (1990) showed

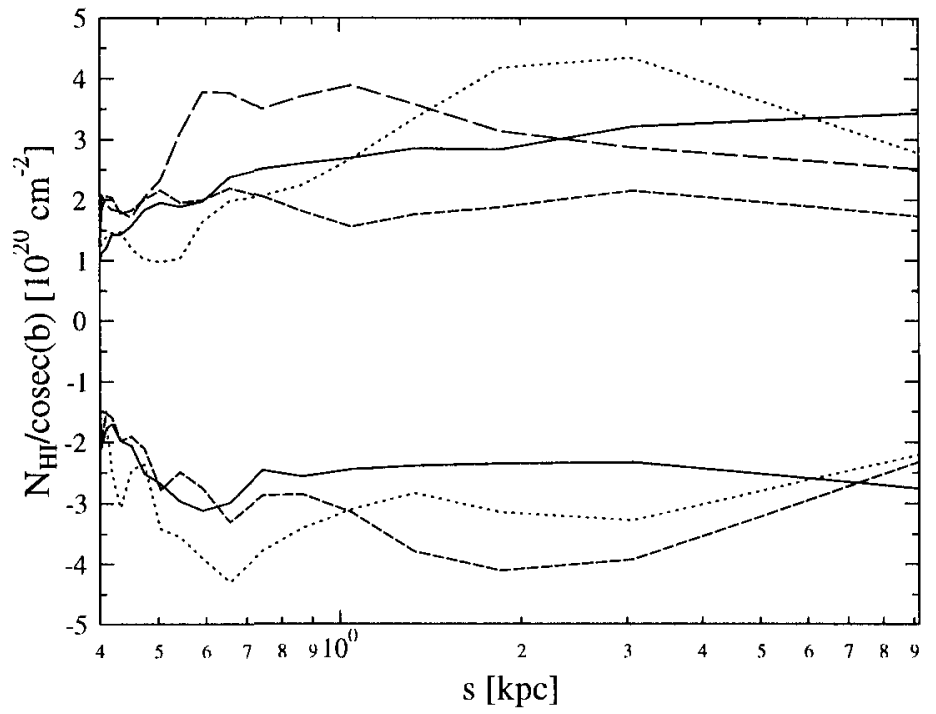

Fig. 1. Vertical projection of the total $\mathrm{H}$ I column density, $N_{\mathrm{HI}} \times \sin (b)$, versus the path length $s$ through the Galactic layer, $\frac{z_{0}}{\sin (b)}$, for $z_{0}=400 \mathrm{pc}$. Positive latitudes for $N_{\mathrm{HI}} \geq 0$, negative latitudes for $N_{\mathrm{HI}}<0$. Solid, dotted, dashed and long dashed lines indicate the first, second, third and fourth Galactic quadrant, respectively. The low values of $N_{\mathrm{HI}} \times \sin (b)$ at small path lengths, $s$, are indicative of the reduced column densities of the local void.

that this depression can be modelled by assuming that in a constant density environment $\mathrm{H}$ I gas is swept out of the region presently occupied by the local void. The sweeping mechanism may be a supernova blast wave or a strong stellar wind. This is the basic idea of the so-called "displacement" model. If all of the observed soft X-ray emitting plasma is placed in front of the first $N_{\mathrm{HI}} \simeq 10^{19} \mathrm{~cm}^{-2}$ of $\mathrm{H} \mathrm{I}$ column density along the line of sight and if a constant density and temperature is assumed for that plasma, then a length, $R$, of the line of sight through this plasma can be derived for each observed direction from the emission measure of the plasma. One finds an average length of $R \simeq 100 \mathrm{pc}$ for $n_{\mathrm{e}} \simeq 10^{-3} \mathrm{~cm}^{-3}$, consistent with the radius of the local void. 


\section{Clouds Inside the Local Void}

The very local clouds $(d<10 \mathrm{pc}$, say), sometimes called the local fluff, are not discernable in the $\mathrm{H} 121-\mathrm{cm}$ line or any other emission down to sub-mm wavelengths. Therefore the local fluff is not discussed here. In Table 1 we have collected some of the important astrophysical parameters of clouds which appear to be located in the local void. All of the four clouds are seen in $\mathrm{H}$ I and IR emission, one of them (LVC159-34+12, or MBM12) is detected in CO. All clouds, except LVC159-34+12, display a rather filamentary morphology with a ratio of the major to the minor axis of about 10 to 1 . H I column densities are all around $N_{\mathrm{HI}} \simeq 1 \cdot 10^{20} \mathrm{~cm}^{-2}$ with a rather small velocity dispersion implying kinetic temperatures of about $100 \mathrm{~K}$ or less. Distances of all clouds except IVC288-34+4 have been derived in two independent ways. First, interstellar absorption lines in stellar spectra constrains the upper-distance limit of the cloud; details of these distances can be found in the references given in the last line of Table 1. Second, soft $\mathrm{X}$-ray radiation transport considerations can give an independent distance estimate if the X-ray volume emissivity of the plasma in the Local Bubble is assumed to be constant and if its full radius at the position of the cloud is known. The distance of $39 \mathrm{pc}$ listed for LVC 88+36-2 is derived from X-ray shadowing assuming a radius of $100 \mathrm{pc}$ for the Local Bubble in this direction. Interstellar absorption line studies give an upper limit of $60 \mathrm{pc}$. Since the radius of the local void is about $100 \mathrm{pc}$ in this direction, LVC88+36-2 is clearly located inside this void. As for LVC88+362 , it has long been established that LVC159-34+12 (MBM12) is also located inside the local void. For LVC $75+35+5$ Kerp \& Pietz (this volume) argue

Table 1. Properties of some local clouds. The star denotes quantities derived from $\mathrm{CO}$ line observations all others are based on $\mathrm{H}$ I 21-cm line measurements.

\begin{tabular}{lrrrr}
\hline name & LVC & LVC & LVC & IVC \\
& $88+36-2$ & $75+35+5$ & $159-34+12$ & $288-34+4$ \\
type of radiation & $\mathrm{HI}, I R A S$ & $\mathrm{HI}, I R A S$ & $\mathrm{HI}, I R A S$, & $\mathrm{HI}$, IRAS \\
& & $\mathrm{CO}$ & \\
morphology & filamentary & filamentary & cloud & filamentary \\
size: major $\times$ minor axis & $5^{\circ} \times 0.5$ & $15^{\circ} \times 2^{\circ}$ & $1^{\circ} \times 1^{\circ}$ & $25^{\circ} \times 2^{\circ}$ \\
position angle & $45^{\circ}$ & $45^{\circ}$ & & $0^{\circ}$ \\
$\mathrm{H}$ I column density $\left[\mathrm{cm}^{-2}\right]$ & $<1 \cdot 10^{20}$ & $\leq 2 \cdot 10^{20}$ & $\sim 1 \cdot 10^{21}$ & $2 \cdot 10^{20}$ \\
velocity dispersion $\left[\mathrm{km} \mathrm{s}^{-1}\right]$ & 1.0 & $\simeq 1.0$ & & $\sim 1.5$ \\
kinetic temperature, $T_{\mathrm{kin}}[\mathrm{K}]$ & 120 & $\simeq 120$ & $\sim 10$ & 200 \\
distance, $d[\mathrm{pc}]$ & 39 & 50 & 65 & 130 \\
total $\mathrm{H}$ I mass, $M_{\mathrm{HI}}\left[\mathrm{M}_{\odot}\right]$ & 15 & 360 & 30 & 3000 \\
volume density, $n_{\mathrm{HI}}\left[\mathrm{cm}^{-3}\right]$ & 80 & 200 & 1000 & 70 \\
pressure $\frac{P}{k}\left[\mathrm{~K} \mathrm{~cm}{ }^{-3}\right]$ & $1 \cdot 10^{4}$ & $2 \cdot 10^{4}$ & $1 \cdot 10^{4}$ & $1 \cdot 10^{4}$ \\
references & Wennmacher & Kerp \& Pietz & Benjamin & Penprase \\
& 1994 & this volume & et al. 1996 & et al. 1997 \\
\hline
\end{tabular}


from both interstellar absorption lines and from soft X-ray absortion that it is probably located within the local void. IVC288-34+4 may be inside the Local Bubble inspite of its rather large distance of $130 \mathrm{pc}$ because the radius of the bubble is also rather large in this direction.

Using distances, the angular sizes and the observed column densities, the clouds have the total $\mathrm{H}$ I masses given in line 10. Assuming circular symmetry, average volume densities are given in line 11 . The temperature and density given for MBM12 were derived from molecular lines (Magnani et al ApJ $295,402)$. From the kinetic temperatures in line 8 and the volume densities in line 11 one can estimate the pressures given in line 12 . The uncertainties of this estimate for the pressure are enormous. This is so because the line width may be dominated by turbulence or because the filament is a sheet seen edgeon rather than a worm-like structure. Then the density, the temperature and the pressure can be very much smaller. Nevertheless it is striking that the pressures derived here are all pretty similar and fall in the range of pressures which are models of the Galactic interstellar medium (e.g. Wolfire et al 1995).

\section{The Quest for a Wall of the Bubble}

A minimum $\mathrm{H}$ I column density of $N_{\mathrm{HI}} \leq 1 \cdot 10^{20} \mathrm{~cm}^{-2}$ is found in various directions at the high latitude sky. Column densities as low as this represent gas which is in the form of the so-called intercloud medium. It has a velocity dispersion of $\sigma \sim 10 \mathrm{~km} \mathrm{~s}^{-1}$, is rather smoothly distributed on the sky and follows most closely the distribution of a plane-parallel layer (Mebold 1972, Falgarone \& Lequeux 1973). H I in the form of clouds which are immersed in this medium dominates at lower latitudes (say $b<40^{\circ}$ ). In the presence of clouds the column density is typically more than doubled. These structural features imply that the local void is rather porous at high latitudes and may actually be open for dynamical interaction with the Galactic halo.

The most complete review of more than 100 molecular clouds at $|b|>$ $25 \mathrm{deg}$ has recently been published by Magnani, Hartmann \& Speck (1996). From the observed dispersion $\sigma \simeq 5.9 \mathrm{~km} \mathrm{~s}^{-1}$ of the centre velocities of these clouds the authors derive an average $\langle z\rangle=87 \mathrm{pc}$ above the Galactic plane. This corresponds to an average distance of $\langle d\rangle=105 \mathrm{pc}$ which coincides remarkably well with the average radius of the Local Bubble. Due to the lack of precise distance determinations is not possible to check whether the frequency distribution of clouds shows a peak at the radius of the Local Bubble or whether their space density is constant starting outwards at that radius. Therefore the coincidence of $\langle d\rangle$ and the mean radius of the local void is nothing but a confirmation that we have locally a void of neutral interstellar material. From the global average of the centre velocities $\langle v\rangle$ $=-0.3 \mathrm{~km} \mathrm{~s}^{-1}$ one can conclude that there is no significant expansion of these clouds. There is no indication that the concept of an expanding bubble applies to the void around the Sun. 
The distribution of clouds on the sky shows a remarkable absence of molecular clouds at $|b|>60^{\circ}$. This could mean that the polar region is the place of a break-out of the LB into the halo of our Galaxy. If this were so, then we have to explain the fact that we do not see a coherent low column density structure in high Galactic latitudes. Rather we see various clouds or cloud complexes which we do not expect to find within the breakout region of a local Supernova event. This makes the concept of a bubble suspicious, once more.

Finally in this section we like to mention the structure of the ISM in the so-called Hercules Region (Lilienthal et al. 1992). This is a region bounded by $40^{\circ}<l<70^{\circ}$ and $35^{\circ}<b<55^{\circ}$. Distance estimates from interstellar absorption lines of the $\mathrm{HI}$ clouds in this region indicate excessive distance variations (some larger than a factor of two) on angular scales of a few degrees. This implies structures in 3-D space which resemble the finger of God, pointing to you and telling you that you are wrong! Here we can definitely state that a wall of the Local Bubble can not be found!

\section{H I Features Beyond the Local Void}

\subsection{IVCs with Molecular Emission}

There is a class of clouds in high latitudes, the so-called intermediate-velocity clouds (IVCs, $-15>v_{\text {LSR }}>-100 \mathrm{~km} \mathrm{~s}^{-1}$ ), which are most likely located outside the local void and frequently show signs of dynamical interaction with the plasma in the Galactic halo or the interface of the halo and the disk (Wesselius \& Fejes 1973, Tenorio-Tagle \& Bodenheimer 1988, Kuntz \& Danly 1996, Moritz et al. 1997). There are three IVCs which show molecular line emission, IVC 92+38-22, IVC 135+53-45, and IVC 211+63-38.7. The first two of these are closely associated with HVCs, i.e. high latitude $\mathrm{H}$ I clouds at velocities $\left|v_{\mathrm{LSR}}\right|>100 \mathrm{~km} \mathrm{~s}^{-1}$ and thus are candidates for an interaction of the kind specified before.

IVC $135+53-45$ is part of the largest IVC complex in the north galactic hemisphere is the so-called IVC-arch (see Kuntz \& Danly 1996). Interstellar absorption line studies indicate a distance of up to $1.7 \mathrm{kpc}$. IVC $92+38-22$ which is frequently called the Draco Cloud (e.g. Mebold et al. 1985, Herbstmeier et al. 1996) is one of three high latitude molecular clouds which is found at intermediate negative velocities (Magnani, Hartmann \& Speck 1996). Its distance is in dispute with estimates ranging between $300 \mathrm{pc}$ and $800 \mathrm{pc}$ (Lilienthal et al. 1991, Mebold et al. 1985). There are two particularly interesting aspects of IVC 92+38-22: first, it was the first interstellar cloud that revealed an X-ray shadow in the $1 / 4 \mathrm{keV}$ soft X-ray background (Snowden et al. 1991). This can be used to derive the molecular hydrogen content in IVC $92+38-22$. Second, it has a steep velocity gradient perpendicular to 
the boundary facing the Galactic centre. This is indicative of an interaction whose nature is not understood up to now.

Our group has studied the $\mathrm{X}$-ray shadows of IVC $92+38-22$ to derive a new and independent estimate of the so-called X-factor $X=\frac{N\left(\mathrm{H}_{2}\right)}{W C O}$. This ratio is frequently used for the conversion of the ${ }^{12} \mathrm{CO}(1 \rightarrow 0)$ line integral into column densities of molecular hydrogen $\mathrm{H}_{2}$. If this factor were strongly variable through-out the Galaxy, column densities of molecular hydrogen derived from this factor would be very unreliable. From the IRAS $100 \mu \mathrm{m}$ intensities, $I_{100}$, observed $\mathrm{CO}$ - and $\mathrm{HI}$ lines, and the assumption that the $100 \mu \mathrm{m}$ intensity is a linear measure of the total column density of hydrogen nuclei. Herbstmeier et al. (1993) find values in the range $\left(0.02 \cdot 10^{20}<X<0.26\right) \cdot 10^{20} \frac{\mathrm{cm}^{-2}}{\mathrm{Kkm} \mathrm{s}^{-1}}$. This is more than a factor 10 smaller than the standard X-factor of $X=$ $2.4 \cdot 10^{20} \frac{\mathrm{cm}^{-2}}{\mathrm{Kkm} \mathrm{s}^{-1}}$. Moritz et al. (1997) analyze the anti-correlation of the soft $\mathrm{X}$-ray count rate versus observed $\mathrm{H}$ I column density $N_{\mathrm{HI}}$ or $I_{100}$. They find absorbing column densities which are more than twice as large as the observed $N_{\mathrm{HI}}$. At the edge towards low galactic latitudes and longitudes, up to $70 \%$ of the total neutral hydrogen is in molecular form. They also find an FIR emissivity per hydrogen nucleon of about $1.0 \cdot 10^{-20} \mathrm{MJy} \mathrm{sr}^{-1} \mathrm{~cm}^{2}$, which is constant through out the Draco cloud. In contrast, the FIR emissivity per $\mathrm{H}$ I atom varies strongly across the Draco cloud. From these results the authors derive $\mathrm{X}$-factors in the range $(0.17<X<0.52) \frac{\mathrm{cm}^{-2}}{\mathrm{~K} \mathrm{~km} \mathrm{~s}^{-1}}$, values which are larger than those found by Herbstmeier, Heithausen \& Mebold (1993) but which are still much smaller than those found for molecular clouds in the Galactic plane.

Moritz et al. (1997) find that the emission measure of the X-ray gas behind the Draco cloud is about 5 times larger than that of the Local Bubble if plasma temperatures of $T \simeq 10^{6.3} \mathrm{~K}$ and $T \simeq 10^{5.85} \mathrm{~K}$ are adopted for the background and the Local Bubble, respectively. Taking into account that the Draco clouds are located out of the Galactic disk, the large emission measure of the background is unequivocal evidence for a bright Galactic X-ray corona.

The second aspect of particular interest are sharp velocity gradients. In the Draco cloud is a steep velocity gradient of $\simeq 1 \mathrm{~km} \mathrm{~s}^{-1} \operatorname{arcmin}^{-1}$ perpendicular to the steep edge facing the Galactic center direction (see Herbstmeier et al. 1996). This gradient, which could only be found with the high angular resolution provided by $\mathrm{HI}$ aperture synthesis observations with the WSRT and the VLA, shows up consistently for more than $2^{\circ}$ along this edge. It may be caused by ram-pressure decelleration of the Draco cloud while it is penetrating the lower Galactic halo on its way towards the disk. The material exerting the ram pressure is either the intercloud $\mathrm{H}$ I gas located e.g. in the Lockman layer (Dickey \& Lockman 1990) or else the ionized medium of the Reynolds layer (Reynolds 1991). Alternatively this gradient may be caused by a Galactic wind rising from the disk at high speed (e.g. Kahn 1989).

IVC $135+54-45$ is the second of the high latitude intermediate-velocity clouds which shows molecular line emission, is associated with an HVC, and 
shows an X-ray shadow. Benjamin et al. (1996) derive the distance to this cloud complex as $d=(355 \pm 95) \mathrm{pc}$, which corresponds to $|z|=(285 \pm$ 75) pc. Estimating the effects of extinction, the most likely value could be as low as $d=240 \mathrm{pc}$. This distance puts the X-ray emission behind a total estimated atomic mass of $1600 \mathrm{M}_{\odot}$. The molecular mass of the cloud core, $\mathrm{G} 135.3+54.5$, is probably much smaller.

Some evidence for an interaction of $\mathrm{HVC} 133+55-120$ with this IVC has been derived by Weiss (1997) from H I aperture synthesis observations obtained with the WSRT. These data show a very close positional association of IVC and $\mathrm{HVC}$ and an orientation of the filamentary structure of the IVC $\mathrm{H}$ I emission which is suggestive of shocks located in the interface region between IVC and HVC. Also, the HVC shows a velocity gradient at one of its edges which appears to connect the emission of $\mathrm{HVC}$ to the velocity of the IVC. We call this phenomenon "velocity bridge" and interpret it as evidence for a decelleration of the HVC during the course of its approach towards the Galaxy (see below). The nature of the decellerating agent appears rather unclear at the moment.

Recent CO line observations of IVC $135+54-45$ carried out with the Pico Veleta $30-\mathrm{m}$ telescope by Weiss, Mebold \& Heithausen (in prep.) indicate that the $\mathrm{CO}$ line intensities are rather large, i.e. that the so-called $\mathrm{X}$-factor is very small, also for this IVC. If HVC $134+55-120$ and IVC $135+54-45$ do indeed interact, then both must be at essentially the same distance. Since the IVC is at a distance of about $350 \mathrm{pc}$ this means that the $\mathrm{HVC}$ is exceptionally close to the Galactic plane. If the material in the HVC has the same heavy element abundance and the same total gas column density as the IVC then the HVC should show up in absorption as does the IVC. There is as yet no information about the distance of $\mathrm{HVC} 134+55-120$ available. But it is of considerable interest that most IVCs do show IR emission, e.g. in the IRAS 100 and $60 \mu \mathrm{m}$ bands, while HVCs in general do not show any IR emission (Wakker \& Boulanger 1986). This is also true in the cases of the IVCs and HVCs discussed in this section. The absense of IR emission could either be due to the absense of dust or the absence of a radiation field that does the heating of the dust. If HVC $134+55-120$ indeed interacts with IVC $135+54-45$ it must be the lack of dust because the radiation field is obviously strong enough to heat the dust in the IVC. Since interstellar absorption lines of HVCs observed towards bright nuclei of galaxies show only mariginal amounts of heavy elements (e.g. Wakker et al. 1996) the dust content of HVCs remains a critical point of the discussion of the HVC-IVC interaction scenario. If, in particular, HVC $134+55-120$ does contain dust, then we would have to accept the conclusion that the dust is not heated by the Galactic radiation field because the HVC is not close to the Galactic plane. Then, the evidence for an interaction of the HVC and the IVC would be accidental in the present case. 


\subsection{High Latitude $H_{\text {I }}$ Gas with Large Velocity Dispersion}

The indications for an interaction of the IVCs with their ambient medium discussed in the previous subsection obviously require an interaction partner of sufficient density. The densities $n=\left(10^{-2}-1\right) \mathrm{cm}^{-3}$ are much higher than the densities expected from the standard z-distributions for atoms or electrons. Since these distributions predict densities of $n<10^{-3} \mathrm{~cm}^{-3}$ at the typical z-distances of the IVCs of, say $1 \mathrm{kpc}$, we asked ourselves whether or not there is evidence for gaseous constituents of the Galactic halo which have not been noticed up to date.

The Leiden/Dwingeloo H I Survey by Hartmann \& Burton (1997) has been searched by Kalberla et al. (1997) and Westphalen et al. (1997) for H I gas that may be residing in the Galactic halo. These authors searched the high latitude regions of the Galaxy for the presence of $\mathrm{H}$ I emission with very large velocity dispersion. After smoothing the data over areas of $5^{\circ}$ by $5^{\circ}$. they indeed found faint emission $\left(T_{\mathrm{B}} \simeq 0.01 \mathrm{~K}\right)$ with a velocity dispersion of $\sigma \simeq$ $60 \mathrm{~km} \mathrm{~s}^{-1}$ and a column density $N_{\mathrm{HI}} \simeq 2 \cdot 10^{19} \mathrm{~cm}^{-2}$, the so-called "Large Velocity Dispersion" (LVD) component. Assuming hydrostatic equilibrium for the z-distribution of atoms and ions and assuming energy equipartition of gas, plasma, magnetic fields, and cosmic rays, Kalberla, Pietz \& Kerp (this volume) derive a scale high perpendicular to the Galactic plane of $h_{\mathrm{z}} \simeq$ $4.4 \mathrm{kpc}$.

It is plausible that the LVD component is only the neutral fraction of the predominantly ionized plasma in the lower Galactic halo. The nature, in particular the degree of ionisation, of this plasma is not $c$ lear at present. It is likely, however, that the LVD gas is intermixed (see Kalberla, Pietz \& Kerp, this volume) with the plasma emitting the X-rays which have recently been detected in the Galactic halo (e.g. Pietz et al., this volume) and with the plasma that is responsible for the interstellar absorption lines of heavy ions observed in the UV and EUV range of spectrum (de Boer, this volume). If this is true, the neutral gas in the LVD emission component may represent density enhancements, something like density- "icebergs", in an otherwise ionized plasma of lower density. These enhancements may be similar to the $\mathrm{H}$ I features found within the local void (see section 4 ). They merge into a diffuse background when looked at with a $5^{\circ}$ beam. Such a picture may lead us to speculate further that HVCs are nothing but anomalously large ones of these density-icebergs. Also, the enhanced X-ray emission associated with some HVCs (Kerp et al. 1996, Kerp et al., this volume) may be produced in the regions of higher density which pile up in front of an infalling HVC. In these regions the ram pressure of the HVC increases the emission measure of the compressed plasma and this may lead to an enhancement of the Xray emission over what is emitted by the non-compressed plasma. Details of this emission mechanism are investigated and discussed in a recent paper by Lesch et al. (this volume). 
Of course, this is a rather qualitative picture yet. It will require further investigations to substantiate and refine this picture or to replace it by a better one.

\subsection{HVCs and HVC-Bridges}

Finally in this Section we argue about the origin of the strong velocity gradients found recently close to the edges of HVCs by Pietz et al. (1996). These features have been termed "velocity bridges" by these authors. In spite of this term, it is completely open at the moment whether these bridges which show up in velocity space, actually refer to spatial connections between HVCs and any other of the prominent features in the $\mathrm{H}$ I spectra.

Although the first velocity bridges were discovered by Meyerdierks (1992) these bridges could only recently be established as fairly common features in HVCs. A significant measurement of their faint $\left(T_{\mathrm{B}} \simeq 0.2 \mathrm{~K}\right)$ and very wide (half width $W \simeq 100 \mathrm{~km} \mathrm{~s}^{-1}$ ) spectral signatures require observations of very high baseline accuracy and a very low level of confusion by instrumental stray radiation. At the moment this requirement and an adequate coverage of the sky is provided only by the Leiden/Dwingeloo H I Survey (Hartmann \& Burton 1997). So far this survey has not yet been searched fully and systematically for velocity bridges. Pietz et al. (1996) have only searched the area of HVC complex $\mathrm{C}$ and list 11 such features in their Table 1. Herbstmeier et al. (1995) also find velocity bridges in the HVC complex M.

Typical parameters of these bridges are velocity ranges of $\Delta v \simeq 50 \mathrm{~km}$ $\mathrm{s}^{-1}$, the brightness temperatures and half widths are as given above and position shifts between the two ends of the bridges are between 0.5 to $2^{\circ}$. Estimating the energy of the process responsible for producing the bridges from the parameters provided by Pietz et al. (1996) we find an energy content of about $5 \cdot 10^{49} \mathrm{ergs}$. This amounts to a few percent of the total kinetic energy content of the associated HVCs.

The parameters provided by Pietz et al. (1996) also allow us to estimate the time scale over which this energy content is built up. The velocity bridges may be regarded as the result of a mass loss of the corresponding HVCs in the past: The product of the gas mass, $M_{\mathrm{VB}}$, at a certain velocity, $v$, in the velocity bridge times that velocity (in the rest frame of the HVC) is the momentum of that gas with respect to the HVC. If this momentum is divided by the path length, $s$, that the gas has travelled from the $\mathrm{HVC}$ we can calculate the relative mass loss rate of the $\mathrm{HVC}$ from $\left(\frac{\partial M_{\text {HVC }}}{\partial t}\right)$ : $M_{\mathrm{HVC}}=\left(M_{\mathrm{VB}} \cdot v\right):\left(M_{\mathrm{HVC}} \cdot s\right)$. Using $\frac{M_{\mathrm{VB}}}{M_{\mathrm{HVC}}} \simeq 0.05$ from the observations, $v=\frac{v}{2} \simeq 25 \mathrm{~km} \mathrm{~s}^{-1}$, and estimating the path-length from the position shift between the two ends of the bridges of, say $1^{\circ},(s=60 \mathrm{pc})$ and a distance of $3 \mathrm{kpc}$, we get $\left(\frac{\partial M_{\mathrm{HVC}}}{\partial t}\right): M_{\mathrm{HVC}} \sim 7 \cdot 10^{-8} \mathrm{a}^{-1}, \mathrm{c}$ orresponding to a time scale for the observed mass loss of about $\tau \sim 2 \cdot 10^{7} \mathrm{a}$. This estimate is a lower limit to the true time scale because the value of $s$ that we have used 
is smaller than its true value. We can see only the projection $s \times \sin (\alpha)$ of the true path length, $s$, onto the sky. The true path length is probably much larger than the observed projection because selection effects favor the detection of velocity bridges with small $\alpha$. The selection effect which is at work here is due to the fact that the observed brightness temperature of the bridges increases with decreasing $\alpha$ (Mebold et al., in prep.).

Adopting a value of $\tau>3 \cdot 10^{7}$ a, we see that the velocity bridges are rather long-lived phenomena. This long time scale imposes constraints on to the mechanisms that may be producing velocity bridges. Pietz et al. (1996) propose three alternative mechanisms:

1. inelastic collisions of HVCs and IVCs with clouds at velocities close to zero,

2. continous sweeping of HVCs and IVCs by the Reynolds - or the Lockman layers, or

3 . interaction with a Galactic wind. Selecting from these options we propose that slow and continous sweeping of the outer parts of a HVC in the gaseous Galactic halo is the most likely mechanism. A possible scenario which may lead to a decelleration of parts of an HVC in the Galactic environment has been modelled by Benjamin (1997). Considering a HVC as a conglomerate of clouds of different densities the drag force will lead to a spatial seperation of these clouds. Those with low density will stay behind, those higher densities will be in the lead. The free-fall time scale for a typical HVC starting at say $10 \mathrm{kpc}$ under the drag force conditions of a gaseous Galactic halo is of the order of $3 \cdot 10^{7} \mathrm{a}$. This is in agreement with the time scale estimated for the mass loss associated with HVC velocity bridges. It is therefore conceivable that velocity bridges are indeed produced by the drag force of a gaseous Galactic halo on HVCs, which consist of a spectrum of clumps with different densities. Since this requirement is indeed met by HVCs (Wakker 1990) we consider it worth while to a more detailed investigation of the origin of $\mathrm{HVC}$ velocity bridges in the frame of the concept persented here.

All the HVCs that are associated with excess X-ray emission are also associated with velocity bridges. Given the long time scale for producing bridges in the scenario decribed above it is unlikely that the observed X-ray luminosity for HVCs with velocity bridges is being produced by the conversion of their kinetic energy into heat. As stated above it is more likely that the enhanced emission is due an increase of the emission measure caused by the compression of the ambient medium. This means that most of the energy comes from the physical state of the halo plasma and not from the kinetic energy of the HVC. - At any rate, it seems likely that velocity bridges are a test probe for the physical state of the plasma in the Galactic halo that is worth exploiting further. 


\section{Conclusion}

It seems to us that the evidence for a local "bubble" provided by observations in other wavelength regimes, in particular in the soft X-ray band, can also consistently be explained by placing the local void in the interarm region between the Sagittarius and the Perseus spiral arms of our Galaxy. This void would then appear as one of many places in the interarm regions of our Galaxy, a proposition that has previously been put forward by other authors (see Hirth et al. 1991, Jacobsen \& Kahn 1986).

The least well understood phenomenon presented in this paper is the extreme velocity gradients associated with a large fraction of the HVCs, called velocity bridges, and the moderate velocity gradients associated with some IVCs (see section 6.3 ). For IVCs, well defined gradients are found, e.g. for the Draco nebula (Herbstmeier et al. 1996) and for the IVC arc by Kuntz \& Danly (1996). For HVCs these gradients could only be established (Pietz et al. 1996) with the very high sensitivity of the new Leiden/Dwingeloo $\mathrm{H}$ I survey (Hartmann \& Burton 1997) although the first bridges were reported by Meyerdierks in 1992. The nature of these velocity gradients is essentially not understood. Obviously they represent rather energetic phenomena. Among the possible interpretations are inelastic collisions of IVCs and HVCs with Galactic gas at velocities close to zero, continuous sweeping of these clouds during their passage through a teneous medium in the Galactic halo, and interactions with a Galactic wind.

At any rate, HVCs with velocity gradients appear to be very interesting probes of their Galactic or extragalactic environment while the corresponding IVCs probe the interface between the Galactic disk and halo. Both probably hold interesting clues about their astrophysical origin and evolution. Given the importance of these clouds and their origin for problems like primordial gas replanishment and chemical evolution of the Galaxy or the phenomenon of a Galactic fountain, or given the ill understood physical conditions of the plasma and the gas in our halo, it seems very worth while to continue working on the nature of these phenomena.

Acknowledgements. We like to thank all scientists of the Radioastronomisches Institut der Universität Bonn and outside who have contributed to the results presented or discussed in the present paper. The work presented here was supported by DFG under project No. ME 745/17-1(2) und DARA project No.50 OR 9203.

\section{References}

Benjamin R.A. (1997): "The Physics of Galactic Halos" eds. Lesch et al., AkademieVerlag, p. 33

Benjamin R.A., Venn K.A., Hiltgen D.D., Sneden C. (1996): ApJ 464, 836

Bowyer C.S., Field G.B. \& Mack J.E. (1968): Nat 217, 32

Dickey J. \& Lockman F.J. (1990): ARAA 28, 215 
Falgarone E. \& Lequeux J, (1973): A\&A 25, 253

Frisch P.C. \&, York D.G. (1983): ApJ 271, L49

Hartmann Dap \& Burton W.B. (1997): "Atlas of Galactic Neutral Hydrogen", Cambridge University Press

Herbstmeier U., Heithausen A. \& Mebold U. (1993): A\&A 272, 514

Herbstmeier U. et al. (1995): A\&A 298, 606

Herbstmeier U. et al. (1996): A\&AS 117, 497

Hirth W., Mebold U., Dahlem M. \& Müller P. (1991), Ap\&SS 186, 211

Jacobsen P. \& Kahn S.M. (1986): ApJ 309, 682

Kahn F. (1989): "Structure and Dynamics of the Interstellar Medium", eds. Tenorio-Tagle et al. Proc. IAU Coll. No. 120, 474

Kalberla P.M.W. (1997): "The Physics of Galactic Halos" eds. Lesch et al., Akademie-Verlag, p. 3

Kerp J. et al. (1996): A\&A 312, 67

Kuntz K.D. \& Danly L. (1996): ApJ 457, 703

Lilienthal D., Hirth W., Mebold U. \& de Boer K.S. (1992): A\&A 255, 323

Lilienthal D., Wennmacher A., Herbstmeier U. \& Mebold U. (1991): A\&A 250, 150

Magnani L., Hartmann Dap \& Speck B.G. (1996): ApJS 106, 447

McCammon D. \& Sanders W.T. (1990): ARAA 28, 657

McCammon D., Burrows D.N., Sanders W.T. \& Kraushaar W.L. (1983): ApJ 269, 170

Mebold U. (1972): A\&A 19, 13

Mebold U. et al. (1985): A\&A 151, 427

Meyerdierks H. (1992): A\&A 253, 515

Moritz P. et al. (1997): A\&A, submitted

Penprase B.E. (1997): in preparation

Pietz J. et al. (1996), A\&A 308, L37

Reynolds R.J. (1991): IAU Symp. 144, "The disk-halo connection" ed. Bloemen H., p. 67

Snowden S.L., Cox D.P., McCammon D. \& Sanders W.T. (1990): ApJ 354, 211

Snowden S.L., Mebold U., Hirth W., Herbstmeier U. \& Schmitt J.H.M.M. (1991): Sci 252,1529

Tenorio-Tagle G. \& Bodenheimer P. (1988): ARAA 26, 145

Wakker B.P. (1990): Ph.D. thesis, University of Groningen

Wakker B.P. \& Boulanger F. (1986): A\&A 170, 84

Wakker B.P. et al. (1996): ApJ 473, 834

Weiss A. (1997): Diplomarbeit Universität Bonn

Wennmacher A. (1994): Ph.D. thesis University of Bonn

Wesselius P.R. \& Fejes I. (1973): A\&A 24, 15

Westphalen et al. (1997): "The Physics of Galactic Halos" eds. Lesch et al., A kademie-Verlag, p. 3

Wolfire M.G., Hollenbach D., McKee C.F., Tielens A.G.G.M. \& Bakes E.L.O. (1995): ApJ 443, 152 